\title{
The diagnostic utility of immunohistochemistry in distinguishing between epithelioid mesotheliomas and squamous carcinomas of the lung: a comparative study
}

\author{
Nelson G Ordóñez \\ Department of Pathology, The University of Texas MD Anderson Cancer Center, Houston, TX, USA
}

\begin{abstract}
As both mesotheliomas and squamous carcinomas can present a wide variety of morphological patterns, they can on occasion be confused. Recently, some groups of investigators have called attention to the difficulties that sometimes exist in distinguishing between these malignancies and the need to define a panel of markers that can assist in reaching the correct diagnosis. The aim of the present study is to compare the value of the various immunohistochemical markers currently available for the diagnosis of mesothelioma and squamous carcinoma of the lung. A total of $\mathbf{3 0}$ epithelioid pleural mesotheliomas exhibiting a solid or predominantly solid pattern, and 30 nonkeratinizing squamous carcinomas of the lung were investigated for the expression of the following markers: podoplanin, calretinin, mesothelin, WT1, keratin 5/6, keratin 7 , p63, carcinoembryonic antigen (CEA), MOC-31, Ber-EP4, B72.3, BG-8 (Lewis'), leu-M1 (CD15), and thyroid transcription factor-1 (TTF-1). All $30(100 \%)$ of the mesotheliomas reacted for calretinin, mesothelin and keratin $7,93 \%$ each for podoplanin, WT1 and keratin 5/6, 13\% for Ber-EP4, 7\% each for P63, MOC-31 and BG-8, and 0\% for B72.3, CEA, leu-M1 and TTF-1. All $30(100 \%)$ of the squamous carcinomas were positive for p63 and keratin $5 / 6,97 \%$ for MOC-31, $87 \%$ for Ber-EP4, $80 \%$ for BG-8, 77\% for CEA, $57 \%$ for keratin $7,40 \%$ for calretinin and $\mathrm{B} 72.3,30 \%$ for leu-M1, $27 \%$ for mesothelin, $15 \%$ for podoplanin, and $0 \%$ for WT 1 and TTF-1. After analyzing the results, it is concluded that from a practical point-of-view, a combination of two positive mesothelioma markers (WT1 and calretinin or mesothelin) with two negative mesothelioma markers (p63 and MOC-31) would allow the differential diagnosis to be established between epithelioid mesotheliomas and squamous carcinomas of the lung in nearly all instances.
\end{abstract}

Modern Pathology (2006) 19, 417-428. doi:10.1038/modpathol.3800544; published online 13 January 2006

Keywords: immunohistochemistry; mesothelioma; p63; podoplanin; squamous carcinoma; WT1

An important characteristic of mesotheliomas is their ability to exhibit a broad range of cytomorphologic features and a wide variety of histologic patterns. When they present a tubular or a papillary pattern, they can be confused with adenocarcinomas and when they exhibit a sarcomatoid morphology, they can be confused with sarcomatoid carcinomas, or sarcomas composed of spindle cells or having pleomorphic features. Epithelioid mesotheliomas growing in a solid pattern can be confused with a

Correspondence: Dr NG Ordóñez, MD, Department of Pathology, The University of Texas MD Anderson Cancer Center, 1515 Holcombe Blvd., Houston, TX 77030, USA.

E-mail: nordonez@mdanderson.org

Received 23 September 2005; revised 15 November 2005; accepted 16 November 2005; published online 13 January 2006 variety of non-small-cell carcinomas, including squamous carcinomas. Both squamous carcinomas and mesotheliomas can be composed of large polygonal cells with dense eosinophilic cytoplasm, or clear cells, or they may exhibit a sarcomatoid morphology.

The pleura can be involved by squamous carcinomas either as a direct extension of a primary squamous carcinoma of the lung into the pleural cavity, or as a metastasis from a squamous carcinoma originating in the lung or in an extrapulmonary site. Squamous carcinomas can extensively involve the pleura with encasement of the lung, thus mimicking a mesothelioma both clinically and radiologically, ${ }^{1-3}$ and squamous carcinomas have even been reported in workers exposed to asbestos under the term 'pseudomesotheliomatous' carci- 
noma. ${ }^{1}$ Furthermore, cases of concomitant mesothelioma and squamous carcinoma have been documented. ${ }^{4,5}$ In one instance, a mesothelioma was intimately admixed with a squamous carcinoma of the lung forming a collision tumor. ${ }^{4}$

Over the past two decades, numerous studies have been published on the value of immunohistochemistry as an ancillary technique in the diagnosis of mesotheliomas and there is general agreement that this method is extremely useful in the diagnosis of these tumors. The primary focus of the large majority of these investigations, however, has been on the distinction between epithelioid mesotheliomas and adenocarcinomas, especially those originating in the lung, but very little has been published comparing the immunoprofiles of epithelioid mesotheliomas and squamous carcinomas. Additionally, several groups of investigators ${ }^{3,6}$ have called attention to the difficulties that sometimes exist in distinguishing between the latter malignancies and the need to define the panel of markers that can assist in this differential diagnosis. The purpose of the present study is to determine whether the various markers that are currently used to discriminate between epithelioid pleural mesotheliomas and lung adenocarcinomas have the same diagnostic value in distinguishing between epithelioid mesotheliomas and squamous carcinomas of the lung. Particular emphasis will be placed on those markers that have recently become available and for which there is evidence that they could assist in the diagnosis of mesotheliomas. To my knowledge, a comprehensive study comparing the immunoprofiles of epithelioid pleural mesotheliomas and squamous carcinomas of the lung has not yet been published.

\section{Materials and methods}

The material used in this study was obtained from the files of the Department of Pathology at the University of Texas MD Anderson Cancer Center. It consisted of 30 epithelioid mesotheliomas and 30 squamous carcinomas of the lung. As the mesotheliomas that are the most likely to be confused with squamous carcinomas are those presenting a solid pattern, all of the mesotheliomas selected for this study exhibited a solid or a predominantly solid morphology. The diagnosis of mesothelioma was made using WHO criteria on hematoxylin and eosin stained sections combined with immunohistochemical, ultrastructural, and clinical features. ${ }^{7}$ All of the squamous carcinomas selected for the study were nonkeratinizing squamous carcinomas of the lung since these are the tumors that are most likely to be confused with mesotheliomas. Immunohistochemical studies were carried out on formalin-fixed, paraffin-embedded tissue sections using the avidin-biotin-peroxidase complex method in a Dako AutoStainer (Carpinteria, CA, USA). The primary antibodies that were used are listed in Table 1. The immunostaining was carried out using the LSAB2 peroxidase kit (Dako). To enhance the immunostaining, a heat epitope retrieval procedure was performed using a Black-and-Decker (Shelton, CT, USA) vegetable steamer as previously described. Depending on the antibody, the buffer solutions used were either sodium citrate buffer, $\mathrm{pH}$ 6.0, or a 10:1 solution of Tris-EDTA buffer, $\mathrm{pH}$ 8.0. Enzymatic pretreatment with $0.2 \%$ protease, type XXIV, (Sigma Chemical, St Louis, MO, USA) in TrisEDTA buffer saline, $\mathrm{pH}$ 7.3, at room temperature for $2 \mathrm{~min}$, was used with the Ber-EP4 antibody. The antigen-antibody reaction was visualized using 3-amino-9-ethylcarbazole or 3,3'-diaminobenzidine tetrahydrochloride as chromogen. To evaluate the specificity of the antibodies, known positive and negative tissues were used as controls. The immunostaining was graded on a sliding scale of $1+$ to $4+$ according to the percentage of reactive cells (trace, $<1 \%$; $1+, 1-25 \%$; $2+, 26-50 \% ; 3+, 51-$ $75 \% ; 4+, 76-100 \%)$.

\section{Results}

\section{Immunohistochemical Findings}

The immunohistochemical results are summarized in Table 2.

\section{Podoplanin}

Of the 30 mesotheliomas, $28(93 \%)$ were positive for podoplanin. In eight of these cases, the staining was graded as $4+$, in 10 as $3+$, in six as $2+$, and in four as $1+$. In the better differentiated tumors and in those exhibiting papillary features, the reaction was characterized by a continuous membranous reactivity along the apical cell membranes (Figure 1a). In the solid and less differentiated tumors, the reaction was less consistent, at times appearing to encircle the cells or in other instances, exhibiting a discontinuous staining pattern (Figure 1b). On occasion, intracytoplasmic, globoid-like positivity was seen. Ultrastructural studies demonstrated that this pattern of positivity was associated with the presence of small intracytoplasmic lumens. A total of $15(50 \%)$ of the 30 squamous carcinomas were also podoplanin positive. The positivity usually occurred in the outer cells of the neoplastic nests and the staining pattern was primarily membranous and often occurred along the entire surface of the cells (Figure 1c). In one case, however, the staining was more diffuse and often involved the entire tumor nest. The reactivity was graded as $3+$ in one case, $2+$ in four, and $1+$ in 10 .

\section{Calretinin}

All $30(100 \%)$ mesotheliomas reacted for calretinin. In all instances, the staining was strong and diffuse $(3+$ or $4+)$ and occurred in both the 
Table 1 Antibodies used in this study

\begin{tabular}{|c|c|c|c|c|}
\hline Marker & Source & Type & Dilution & Antigen retrieval \\
\hline Ber-EP4 & $\begin{array}{l}\text { Dako Corporation } \\
\text { (Carpinteria, CA, USA) }\end{array}$ & $\mathrm{MAb}$ & $1: 30$ & Yes (enzymatic digestion) \\
\hline Calretinin & $\begin{array}{l}\text { Zymed } \\
\text { (South San Francisco, CA, USA) }\end{array}$ & PAb (rabbit) & $1: 20$ & Yes (citrate) \\
\hline CD15 & $\begin{array}{l}\text { Becton-Dickinson } \\
\text { (Mountainview, CA, USA) }\end{array}$ & Leu-M1 MAb & $1: 40$ & Yes (Tris-EDTA) \\
\hline CEA & $\begin{array}{l}\text { NeoMarkers } \\
\text { (Fremont, CA, USA) }\end{array}$ & PAb (rabbit) & $1: 175$ & No \\
\hline Keratin $5 / 6$ & Dako Corporation & D5/16B4 MAb & $1: 100$ & Yes (citrate) \\
\hline Keratin 7 & Dako Corporation & OV-TL 12/30 MAb & $1: 100$ & Yes (enzymatic digestion) \\
\hline Lewis $^{y}$ & $\begin{array}{l}\text { Signet Laboratories } \\
\text { (Dedham, MA, USA) }\end{array}$ & BG-8 MAb & $1: 50$ & Yes (citrate) \\
\hline Mesothelin & $\begin{array}{l}\text { Novocastra } \\
\text { (Newcastle-upon-Tyne, UK) }\end{array}$ & 5B2 MAb & $1: 30$ & Yes (Tris-EDTA) \\
\hline $\begin{array}{l}\text { MOC-31 } \\
\text { p63 }\end{array}$ & $\begin{array}{l}\text { Dako Corporation } \\
\text { Santa Cruz Biotechnology } \\
\text { (Santa Cruz, CA, USA) }\end{array}$ & $\begin{array}{l}\text { MAb } \\
4 \mathrm{~A} 4 \mathrm{MAb}\end{array}$ & $\begin{array}{l}1: 50 \\
1: 200\end{array}$ & $\begin{array}{l}\text { Yes (citrate) } \\
\text { Yes (Tris-EDTA) }\end{array}$ \\
\hline $\begin{array}{l}\text { Podoplanin } \\
\text { TAG-72 }\end{array}$ & $\begin{array}{l}\text { Signet Laboratories } \\
\text { BioGenex } \\
\text { (San Ramon, CA, USA) }\end{array}$ & $\begin{array}{l}\text { D2-40 MAb } \\
\text { B72.3 MAb }\end{array}$ & $\begin{array}{l}1: 25 \\
1: 300\end{array}$ & $\begin{array}{l}\text { Yes (Tris-EDTA) } \\
\text { No }\end{array}$ \\
\hline $\begin{array}{l}\text { TTF-1 } \\
\text { WT1 }\end{array}$ & $\begin{array}{l}\text { Dako Corporation } \\
\text { Dako Corporation }\end{array}$ & $\begin{array}{l}\text { 8G7G3/1 MAb } \\
6 \mathrm{~F}-\mathrm{H} 2 \mathrm{MAb}\end{array}$ & $\begin{array}{l}1: 25 \\
1: 40\end{array}$ & $\begin{array}{l}\text { Yes (citrate) } \\
\text { Yes (Tris-EDTA) }\end{array}$ \\
\hline
\end{tabular}

$\mathrm{CEA}=$ carcinoembryonic antigen; TTF-1 = thyroid transcription factor-1; MAb = monoclonal antibody; Pab = polyclonal antibody.

Table 2 Immunohistochemical results

\begin{tabular}{|c|c|c|c|c|c|c|c|c|c|c|c|c|c|c|}
\hline \multirow[t]{3}{*}{ Marker } & \multicolumn{7}{|c|}{ Epithelioid mesotheliomas } & \multicolumn{7}{|c|}{ Squamous carcinomas } \\
\hline & \multicolumn{2}{|c|}{$(\mathrm{n}=30)$} & \multicolumn{5}{|c|}{ Grade of reactivity } & \multicolumn{2}{|c|}{$(\mathrm{n}=30)$} & \multicolumn{5}{|c|}{ Grade of reactivity } \\
\hline & + Cases & $(\%)$ & Trace & $1+$ & $2+$ & $3+$ & $4+$ & + Cases & $(\%)$ & Trace & $1+$ & $2+$ & $3+$ & $4+$ \\
\hline Podoplanin & 28 & (93) & 0 & 4 & 6 & 10 & 8 & 15 & (50) & 0 & 10 & 4 & 1 & 0 \\
\hline Calretinin & 30 & (100) & 0 & 0 & 0 & 8 & 22 & 12 & $(40)$ & 1 & 5 & 3 & 3 & 0 \\
\hline Keratin 5/6 & 28 & (93) & 0 & 3 & 8 & 9 & 8 & 30 & (100) & 0 & 4 & 7 & 7 & 12 \\
\hline Keratin 7 & 30 & $(100)$ & 0 & 0 & 1 & 4 & 25 & 17 & $(57)$ & 2 & 3 & 5 & 4 & 3 \\
\hline Mesothelin & 30 & (100) & 0 & 0 & 4 & 7 & 19 & 8 & (27) & 2 & 2 & 3 & 1 & 0 \\
\hline WT1 & 28 & (93) & 0 & 2 & 5 & 11 & 10 & 0 & $(0)$ & 0 & 0 & 0 & 0 & 0 \\
\hline MOC-31 & 2 & (7) & 1 & 1 & 0 & 0 & 0 & 29 & (97) & 1 & 6 & 2 & 5 & 15 \\
\hline Ber-EP4 & 4 & (13) & 2 & 2 & 0 & 0 & 0 & 26 & (87) & 0 & 7 & 6 & 8 & 5 \\
\hline B72.3 (TAG-72) & 0 & (0) & 0 & 0 & 0 & 0 & 0 & 12 & $(40)$ & 7 & 5 & 0 & 0 & 0 \\
\hline BG-8 (Lewis ${ }^{\mathrm{y}}$ ) & 2 & (7) & 1 & 1 & 0 & 0 & 0 & 24 & (80) & 0 & 6 & 9 & 5 & 4 \\
\hline Leu-M1 (CD15) & 0 & $(0)$ & 0 & 0 & 0 & 0 & 0 & 9 & (30) & 0 & 5 & 4 & 0 & 0 \\
\hline p63 & 2 & (7) & 0 & 0 & 1 & 1 & 0 & 30 & (100) & 0 & 0 & 0 & 5 & 25 \\
\hline CEA & 0 & (0) & 0 & 0 & 0 & 0 & 0 & 23 & (77) & 0 & 7 & 6 & 4 & 6 \\
\hline TTF-1 & 0 & (0) & 0 & 0 & 0 & 0 & 0 & 0 & (0) & 0 & 0 & 0 & 0 & 0 \\
\hline
\end{tabular}

nucleus and the cytoplasm (Figure 1d). In all, $12(40 \%)$ of the squamous carcinomas were also calretinin positive. In three of the cases, the reaction was graded as $3+$, in three $2+$, in five $1+$, and trace in the remaining case. The reaction was both nuclear and cytoplasmic, which is similar to that seen in the mesotheliomas (Figure 1e). 

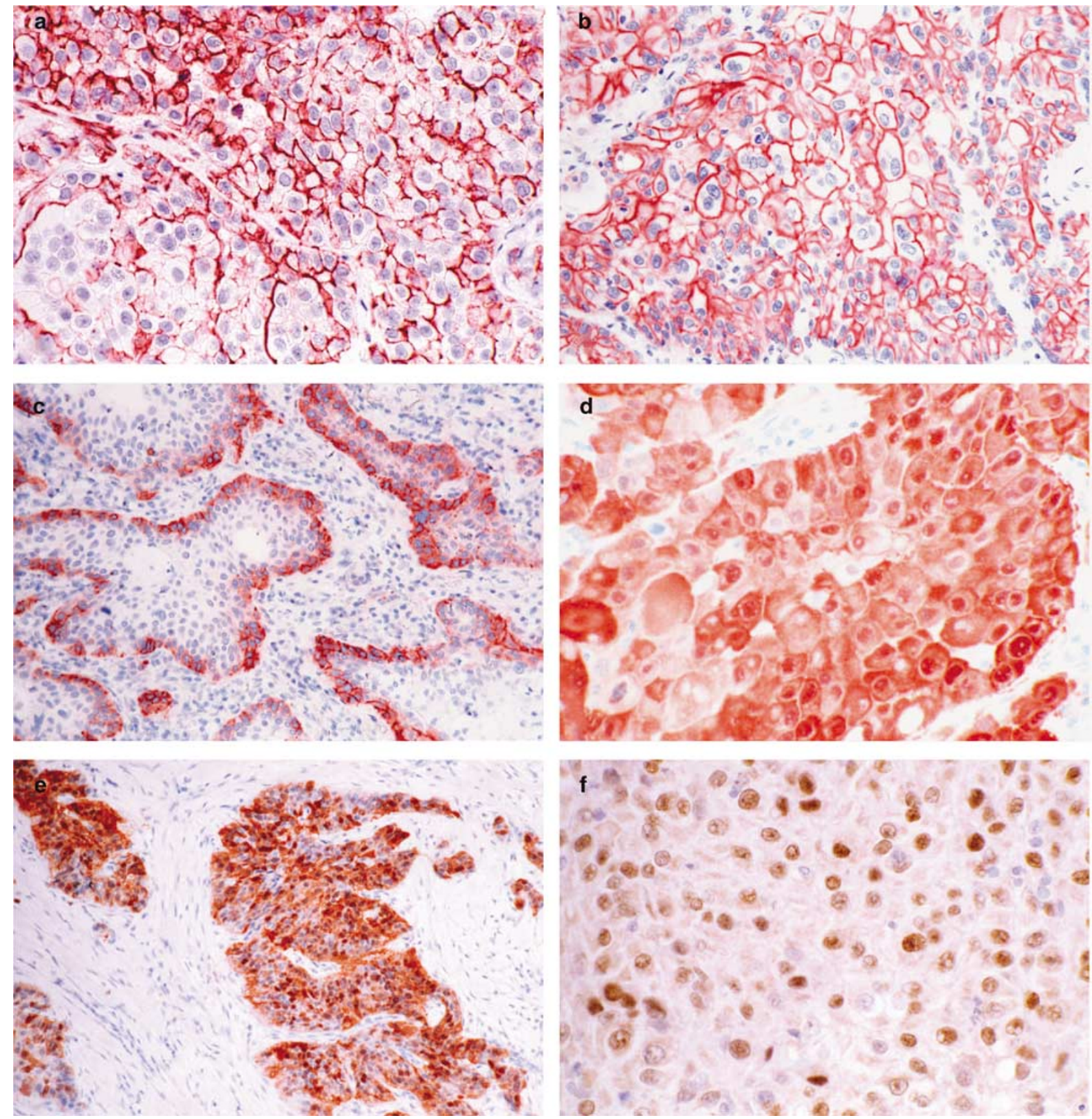

Figure 1 (a) The strong podoplanin reactivity seen along the apical surface of the cells in this mesothelioma discloses a poorly formed papillary pattern which was not apparent in the hematoxylin and eosin stained preparation. (b) Solid area of an epithelioid mesothelioma showing a continuous reaction for podoplanin along the entire cell membrane. (c) Squamous carcinoma demonstrating podoplanin expression in the cells located at the periphery of the tumor nests. (d) Mesothelioma showing strong nuclear and cytoplasmic positivity for calretinin. (e) Squamous carcinoma strongly reacting for calretinin. (f) Mesothelioma showing nuclear WT1 positivity.

Wilms' tumor 1 (WT1) protein

WT1 positivity was seen in $28(93 \%)$ of the 30 mesotheliomas. In most of the cases, the staining was strong and diffuse $(3+$ or $4+)$ and was confined to the nuclei (Figure 1f). All of the squamous carcinomas were negative for this marker.

\section{Mesothelin}

All $30(100 \%)$ mesotheliomas were positive for mesothelin. The reaction was usually strong and diffuse, and it was characterized by a thick, membranous staining pattern. Positivity was also seen on the limiting membrane of the intracytoplasmic lumens when they were present (Figure 2a). 

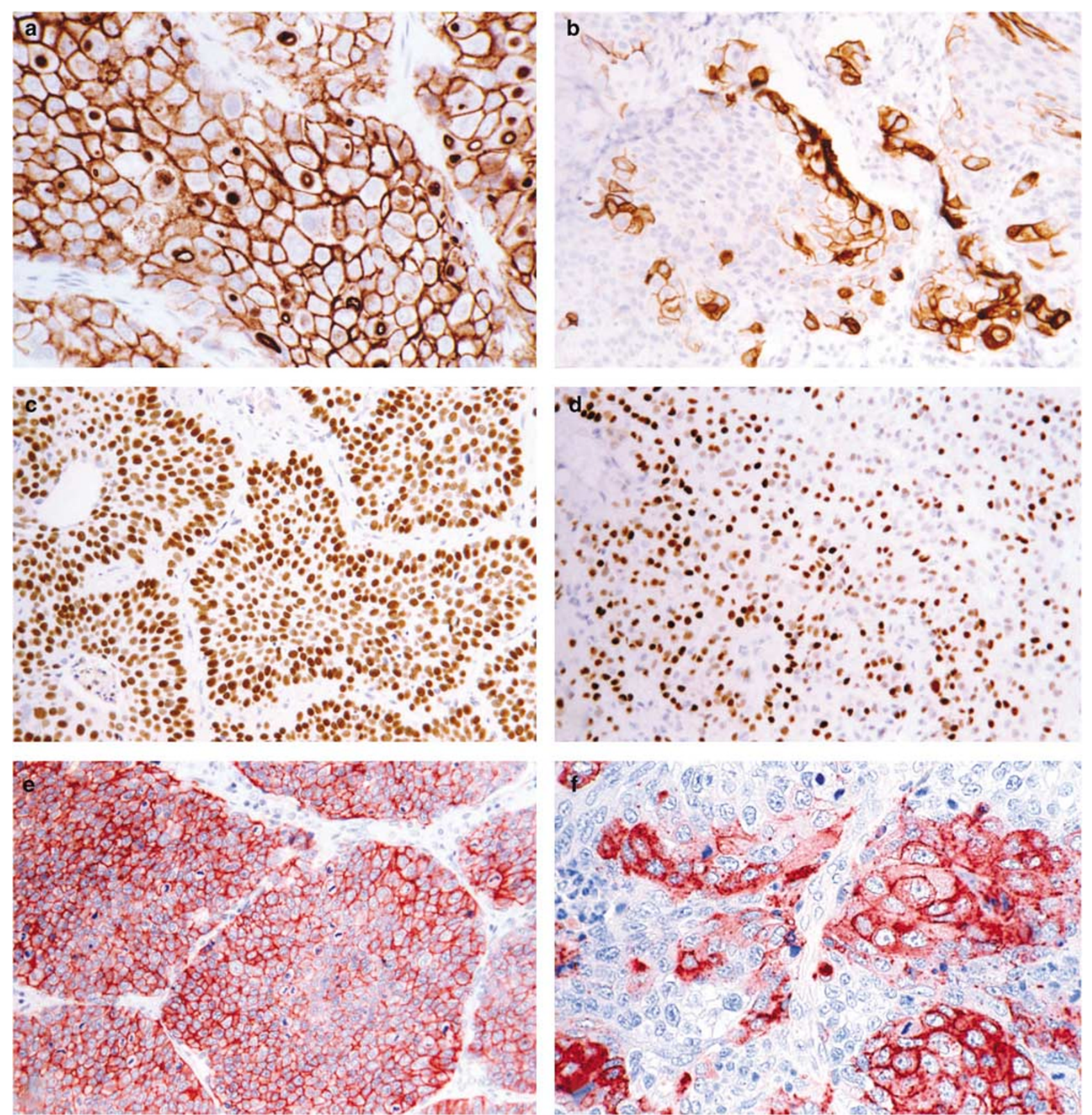

Figure 2 (a) Mesothelioma showing strong reactivity for mesothelin along the cell membrane and on the borders of several small intracytoplasmic lumens. (b) Area of focal mesothelin positivity in a squamous carcinoma. (c) Squamous carcinoma displaying strong nuclear positivity for p63. (d) Same mesothelioma as seen in Figure 1a showing p63 expression. (e) Squamous carcinoma exhibiting strong reactivity with the Ber-EP4 antibody. (f) BG-8 positivity in a squamous carcinoma.

Eight $(27 \%)$ of the squamous carcinomas exhibited mesothelin positivity and in most instances, the staining was focal (Figure 2b). The reaction occurred in over $50 \%$ of the cells $(3+)$ in only one case.

\section{p63}

All $30(100 \%)$ of the squamous carcinomas were p63 positive and in all of the cases, the reaction was nuclear, strong and diffuse (Figure 2c). Only two of the 30 mesotheliomas were p63 positive. In one case, the reaction was graded as $3+$ and in the other, as $2+$ (Figure 2d).

\section{MOC-31}

In all, $29(97 \%)$ of the 30 squamous carcinomas of the lung exhibited MOC-31 reactivity. In most cases, the staining was strong and occurred in the cytoplasm and along the cell membrane. Only two of the mesotheliomas reacted with this antibody and 
in both of these cases, the staining was limited to a few scattered cells.

\section{Ber-EP4}

Of the 30 squamous carcinomas, $26(87 \%)$ stained with the Ber-EP4 antibody. In 13 of the cases, the staining was strong and diffuse $(3+$ or $4+)$ and occurred in both the cytoplasm and cell membrane (Figure 2e). Four (13\%) of the mesotheliomas exhibited Ber-EP4 positivity in a limited number of cells. The staining was graded as $1+$ in two cases, and trace in the remaining two.

\section{$B G-8$}

A total of $24(80 \%)$ of the 30 squamous carcinomas of the lung stained with the BG-8 antibody. In nine cases, the reaction was strong and diffuse and occurred in both the cytoplasm and cell membrane (Figure 2f). Only two (7\%) of the mesotheliomas exhibited BG-8 and in these cases, the staining was limited to a few cells.

\section{Leu-M1 (CD15)}

Only nine $(30 \%)$ of the 30 squamous carcinomas stained with the leu-M1 antibody. In all instances, the staining was focal $(1+$ or $2+)$. None of the mesothelioma cases reacted with this antibody.

\section{Carcinoembryonic antigen (CEA)}

Of the 30 squamous carcinomas, 23 (77\%) exhibited CEA reactivity. The staining was cytoplasmic with accentuation of the reaction along the cell membrane. None of the mesotheliomas were positive for this marker.

\section{B72.3}

In all, $12(12 \%)$ of the 30 squamous carcinomas of the lung reacted with the B72.3. antibody. The reactivity was focal and usually confined to small areas of the tumor. In five of the cases, the reaction was graded as $1+$, and trace in the remaining seven. No reactivity was observed in any of the mesotheliomas.

\section{Keratin 5/6}

Of the 30 mesotheliomas, 28 (93\%) exhibited keratin $5 / 6$ expression. The staining was strong $(3+$ or $4+)$ and evenly distributed throughout the cytoplasm in 17 of these cases. Keratin 5/6 positivity was observed in all $30(100 \%)$ of the squamous carcinomas.

\section{Keratin 7}

All $30(100 \%)$ of the mesotheliomas stained for keratin 7 and the reaction was usually strong and diffuse. In all, $17(57 \%)$ of the 30 squamous carcinomas were keratin 7 positive. In three cases, the reaction was graded as $4+$, in four as $3+$, in five as $2+$, in three as $1+$, and as trace in the remaining two.
Thyroid transcription factor-1 (TTF-1)

All of the mesotheliomas and squamous carcinomas were negative for TTF-1.

\section{Discussion}

While it is generally accepted that immunohistochemistry is a useful ancillary technique in the diagnosis of mesothelioma, very little information is available regarding its use in the distinction between these tumors and squamous carcinomas as most of the published studies have focused on differentiating mesotheliomas from lung adenocarcinomas. In my experience, the differential diagnosis between squamous carcinomas and mesotheliomas can, on occasion, be very challenging, particularly when the latter tumors present a solid or predominantly solid morphology, and the tissue sample is limited. It is also important to be aware that some of the markers that have proved to be very useful in discriminating between epithelioid mesotheliomas and adenocarcinomas do not have the same degree of sensitivity and/or specificity for distinguishing between epithelioid mesotheliomas and squamous carcinomas. For example, because keratin 5/6 and thrombomodulin are often expressed in epithelioid mesotheliomas, but not in lung adenocarcinomas, immunostaining for these markers is often used to distinguish between these two malignancies. ${ }^{8}$ However, neither of these markers has any utility in discriminating between epithelioid mesotheliomas and squamous carcinomas since both are also often expressed in squamous carcinomas. ${ }^{6,9}$

Podoplanin and the D2-40 monoclonal antibody that was raised against an unidentified M2A protein derived from germ cell tumors were initially considered to be two different mesothelioma markers. ${ }^{10-12}$ Recent investigations, ${ }^{13}$ however, have determined that D2-40 specifically recognizes podoplanin, a $38 \mathrm{kDa}$ mucin-type transmembrane protein that was first detected on the surface of the rat glomerular epithelial cells (podocytes) and that was found to be linked to the flattening of foot processes in puromycin-induced nephrosis. ${ }^{14} \mathrm{Be}-$ cause podoplanin has been shown to be expressed in epithelioid mesotheliomas, but not in lung adenocarcinomas, it has been proven to be a very useful immunohistochemical marker in discriminating between these malignancies. ${ }^{10-12,15}$ In the present investigation, $93 \%$ of the mesotheliomas showed podoplanin positivity which was strong and diffuse in the majority of the cases. These results are similar to those previously reported using either the D2-40 antibody ${ }^{10,12,15}$ or another commercially available antipodoplanin monoclonal antibody (AngioBio Company). ${ }^{11,12}$ Conflicting results were obtained in the only two studies investigating podoplanin expression in squamous carcinomas of the lung. ${ }^{12,15}$ In one of these investigations, published only in 
abstract form, Sienko et $a l^{15}$ reported weak reactivity in $44(50 \%)$ of 88 squamous carcinomas of the lung, but no details were given regarding the staining pattern. These results are in contrast to those obtained by this author in which no podoplanin expression was demonstrated in 10 squamous carcinomas investigated. ${ }^{12}$ In the present study, podoplanin expression was shown in $50 \%$ of the cases and, with the exception of one case, the staining was focal and typically occurred in the peripheral cells of the tumor nests. This pattern of reactivity appears to be similar to that observed by Schacht et $a l^{13}$ who recently reported podoplanin expression predominantly in the basal layer of the large majority of their squamous carcinomas of the skin. Since in the current study this distinctive staining pattern only occurred in squamous carcinomas, when present, it may have some utility in distinguishing these tumors from mesotheliomas in which the reaction is frequently diffuse and the exclusively basal distribution is not seen. Additionally, podoplanin immunostaining in mesotheliomas may disclose a papillary pattern in the solid areas of the tumor that is not apparent on hematoxylin and eosin stained preparations; however, this staining pattern is absent in squamous carcinomas.

Calretinin is one of the first of the so-called 'positive' mesothelioma markers that was found to be useful in the diagnosis of mesothelioma. ${ }^{16,17}$ In 1996, Doglioni et $a l^{16}$ reported strong calretinin expression in all 36 epithelioid mesotheliomas, but only focal staining was found in $28(10 \%)$ of 294 adenocarcinomas of various origins and in 10 (18\%) of 55 squamous carcinomas also of various origins. While many subsequent investigations have confirmed the usefulness of calretinin immunostaining in the differential diagnosis between mesotheliomas and lung adenocarcinomas, ${ }^{8,18-29}$ only a relatively few studies have been published on the expression of this marker in squamous carcinomas of the lung. ${ }^{3,6,26,30}$ In the present investigation, all of the mesotheliomas exhibited diffuse strong positivity for calretinin, thus confirming the findings of previous studies..$^{8,16,18,22,25,26,31}$ A total of $12(40 \%)$ of the squamous carcinomas in the current study exhibited calretinin positivity and even though the staining was focal in most instances, in three of the cases, it was strong and diffuse.

WT1 protein is one of the recently recognized positive mesothelioma markers that, because it is frequently expressed in epithelioid mesotheliomas but not in lung adenocarcinomas, has been found to be useful in discriminating between these malignancies. ${ }^{8,32,33}$ WT1 positivity was observed in 28 $(93 \%)$ of the epithelioid mesotheliomas in the present investigation, thus confirming the results of previous studies that this marker is often expressed in these tumors. ${ }^{31-36}$ None of the squamous carcinomas, however, exhibited WT1 expression which is in agreement with the results reported by others indicating that non-small-cell carcinomas of the lung, including squamous carcinomas, are negative for this marker. ${ }^{34,35,37}$

Mesothelin is a cell surface glycoprotein of unknown function that was first described as the antigenic target of the $\mathrm{K} 1$ monoclonal antibody. ${ }^{38} \mathrm{In}$ 1992 using this antibody, Chang et $a l^{39}$ reported strong reactivity in all of their epithelioid mesotheliomas, but in none of the lung adenocarcinomas and, consequently, they suggested that this marker could assist in the differential diagnosis between these malignancies. These observations, however, were not confirmed by more recent investigations using the newly available 5B2 antimesothelin antibody. ${ }^{6,40}$ In the present study, mesothelin expression was demonstrated in all epithelioid mesotheliomas and in eight $(27 \%)$ squamous carcinomas. While mesothelin is not specific for discriminating between these malignancies, some differences were observed in the staining patterns of these tumors. In the mesotheliomas, the pattern was usually membranous, strong, and diffuse, but when staining occurred in the squamous carcinomas, it was focal and more variable. Additionally, mesothelin immunostaining often disclosed the presence of intracytoplasmic lumens in mesotheliomas, but not in squamous carcinomas.

p63 is a recently characterized nuclear transcription factor that shares a high structural homology with p53. ${ }^{41}$ Its gene, located on $3 \mathrm{q} 27$, encodes at least six different isoforms that are divided into those that share transactivating functions with p53 and those that suppress these functions. The latter group of p63 molecules is thought to help maintain the stem/basal cell population and is found in the basal (stem) cells of the stratified epithelia, hair follicles, skin, and prostate, and in myoepithelial cells of the salivary gland and breast. ${ }^{42}$ Expression of p63 has been investigated in a variety of tumors, and it has been found that the p63 gene is amplified and the protein is overexpressed in squamous carcinomas of various primary sites. Most of the studies that have investigated p63 expression in tumors used the 4A4 antibody that detects all p63 isoforms by Western blotting. In the present investigation using the 4A4 antibody, strong p63 positivity was demonstrated in all of the squamous carcinomas. These results are in agreement with those previously reported in the literature in which expression for this protein occurred in $80-100 \%$ of the squamous carcinomas of the lung. ${ }^{43-47}$ Only two studies, with relatively few cases, have investigated the expression of p63 in mesotheliomas and in neither did any of the cases exhibit positivity for this protein. ${ }^{44,48}$ In the present study, two (7\%) of the mesotheliomas expressed p63. This finding indicates that, although uncommon, p63 expression can occur in these tumors, but when present, the percentage of positive cells is usually lower than that seen in squamous carcinomas. It should also be mentioned that p63 has also been reported in $12-33 \%$ of the lung adenocarcinomas, ${ }^{43,45,49}$ a finding which indicates 
that p63 immunostaining has no utility in distinguishing these tumors from mesotheliomas.

MOC-31 is a monoclonal antibody that recognizes an epithelial cell adhesion molecule (Ep-CAM), also known as human pan-carcinoma-associated epithelial glycoprotein-2. Since this antibody reacts with most carcinomas, but only rarely and patchily with epithelioid mesotheliomas, at present, it is considered to be one of the most sensitive and specific of the so-called 'negative' mesothelioma markers for distinguishing between epithelioid mesotheliomas and adenocarcinomas. ${ }^{8}$ The percentage of MOC-31 reactivity reported in lung adenocarcinomas has ranged from 90 to $100 \%$ of the cases and in most instances the staining was described as being strong and diffuse. ${ }^{8}$ This is in contrast to mesotheliomas in which MOC-31 reactivity has been reported in $<10 \%$ of the cases and the staining was limited to a few cells or to small focal areas of the tumor. ${ }^{8,21,50,51}$ Only a few studies with a limited number of cases have investigated the reactivity of this antibody with squamous carcinomas of the lung. ${ }^{8,52}$ In one of these studies, ${ }^{8}$ MOC-31 positivity was reported in all six (100\%) of the squamous carcinomas investigated. In the present investigation, 29 (97\%) of the squamous carcinomas, but only two $(7 \%)$ of the mesotheliomas, stained with this antibody. While the reaction was usually strong and diffuse in the squamous carcinomas, in the mesotheliomas, it was limited to a few tumor cells. These results indicate that this marker could assist in distinguishing between these tumors.

Ber-EP4 is a monoclonal antibody that, like MOC31, also recognizes the Ep-CAM molecule. ${ }^{53}$ The percentage of Ber-EP4 reactivity reported in lung adenocarcinomas has ranged from 96 to $100 \%, 8,21,22$ and from 0 to $35^{8,22,25,54,55}$ in epithelioid mesotheliomas. Only a few studies have been published on the reactivity of Ber-EP4 in squamous carcinomas of the lung and the percentage of positivity has ranged from 29 to $100 \%$ of the cases. ${ }^{3,54-56}$ In the present investigation, $87 \%$ of the squamous carcinomas and $13 \%$ of the mesotheliomas reacted with Ber-EP4. Since the reactivity in squamous carcinomas was often strong and diffuse, in contrast to that seen in the mesotheliomas which, when it occurred, was usually limited to small focal areas of the tumor, BerEP4 could be useful in assisting in distinguishing between these tumors.

BG-8 is a monoclonal antibody that recognizes the blood group Lewis ${ }^{\mathrm{y}}$. The percentage of BG-8 positivity reported in lung adenocarcinomas has ranged from 89 to $100 \%$ and in mesotheliomas from 6 to $23 \% .^{8,33,57,58}$ Only one study has investigated the reactivity of the BG-8 antibody in squamous carcinomas of the lung. ${ }^{59}$ In that study, $10(83 \%)$ of the 12 squamous carcinomas were reported as being BG-8 positive. In the present study, $24(80 \%)$ of the squamous carcinomas, but only two $(7 \%)$ of the mesotheliomas, reacted with this antibody and in contrast to the often strong staining seen in the squamous carcinomas, in the mesotheliomas, it was limited to a few cells.

Since the report by Sheibani et $a l^{60}$ in 1986 indicating that leu-M1 (CD15) was a useful immunohistochemical marker in the diagnosis of mesotheliomas, leu-M1 immunostaining has often been used in the differential diagnosis between mesotheliomas and lung adenocarcinomas. Only a few studies, however, have investigated the expression of this marker in squamous carcinomas of the lung. ${ }^{3,56,61,62}$ In the present investigation, nine $(30 \%)$ of the squamous carcinomas and none of the mesotheliomas were positive for leu-M1. These findings indicate that, because of its low sensitivity, this marker has no practical utility in distinguishing between these two malignancies.

CEA is one of the markers that is most often used in distinguishing mesotheliomas from lung adenocarcinomas. In the present study, $23(77 \%)$ of the squamous carcinomas, but none of the mesotheliomas expressed this marker. These findings confirm previous observations indicating that CEA is often expressed in squamous carcinomas of the lung. ${ }^{63}$ Although some earlier studies reported CEA positivity in a relatively high percentage of mesothelioma cases, it is now believed that CEA expression, if it occurs in these tumors, is a rare phenomenon. ${ }^{8}$

B72.3 is one of the earliest negative markers for mesothelioma that proved to be useful in distinguishing between mesotheliomas and adenocarcinomas. The percentage of lung adenocarcinomas that have been reported to react with this antibody has ranged from 75 to $85 \%$ of the cases, whereas, mesotheliomas have usually been negative. ${ }^{8,25}$ In squamous carcinomas of the lung, the reported percentage of B72.3 positivity has ranged from 45 to $84 \%$ of the cases. ${ }^{59,64-66}$ In the present investigation, $12(40 \%)$ of the squamous carcinomas exhibited B72.3 positivity and the staining was usually limited to small areas of the tumor or a few scattered cells. These findings indicate that B72.3 immunostaining, although useful in distinguishing between lung adenocarcinomas and epithelioid mesotheliomas, ${ }^{8}$ has no utility in discriminating mesotheliomas from squamous carcinomas of the lung.

TTF-1 is a nuclear tissue-specific transcription factor that is expressed in normal lung and thyroid, as well as in tumors derived from these organs. ${ }^{67}$ Although there is general agreement that the large majority of lung adenocarcinomas express TTF-1, ${ }^{8,67}$ there is disagreement in the literature regarding the expression of this marker in squamous carcinomas of the lung. In some of the earlier studies, which used a polyclonal antibody against TTF-1, positivity for this protein was reported in $11-23 \%$ of the squamous carcinomas of the lung. ${ }^{68-70}$ Subsequent investigations using the $8 \mathrm{G} 7 \mathrm{G} 3 / 1$ monoclonal antibody, however, have shown that TTF-1 expression is usually absent in these tumors. ${ }^{47,71-75}$ In the present investigation, none of the squamous carcinomas of the lung or the mesotheliomas exhibited TTF-1 
positivity. While these findings indicate that TTF-1 immunostaining is not useful in discriminating between these malignancies, it may have some utility in distinguishing squamous carcinomas from lung adenocarcinomas since this marker is often expressed in the latter tumors. ${ }^{8}$

In the present investigation, all 30 of the squamous carcinomas, and 28 (93\%) of the mesotheliomas were positive for keratin 5/6, thus confirming previous reports indicating that this marker is usually expressed in both of these tumors. It should be emphasized, however, that even though keratin $5 / 6$ does not have any utility in discriminating between these two malignancies, it can be useful in differentiating between squamous carcinomas and lung adenocarcinomas as the latter tumors are rarely positive for this marker. ${ }^{9}$

Although there is general agreement that both mesotheliomas and lung adenocarcinomas usually express keratin 7 , some controversy exists regarding its expression in squamous carcinomas of the lung. While keratin 7 expression has not been demonstrated in squamous carcinomas of the lung in some studies, particularly the earlier ones, ${ }^{76-81}$ recent investigations have reported positivity for this marker in $22-33 \%$ of the cases. ${ }^{59,72,82}$ In the present study, $17(57 \%)$ of the squamous carcinomas showed keratin 7 positivity, a finding which confirms that this keratin peptide can be expressed in squamous carcinomas of the lung and, therefore, immunostaining for this marker is not useful in distinguishing these tumors from either epithelioid mesotheliomas or adenocarcinomas of the lung.

The value of a large number of immunohistochemical markers in distinguishing between epithelioid mesotheliomas and lung adenocarcinomas was recently investigated by this author. ${ }^{5}$ The conclusion of that study was that calretinin, keratin 5/6, and WT1 were the best positive mesothelioma markers, and CEA, MOC-31, Ber-EP4, and B72.3 were the best of the negative mesothelioma markers for assisting in the differential diagnosis between these malignancies. The results of the present investigation demonstrate, however, that some of these markers do not have the same value in discriminating between epithelioid mesotheliomas and squamous carcinomas of the lung. Since WT1 was found to be expressed in $90 \%$ of the mesotheliomas, but in none of the squamous carcinomas, this marker appears to be the best of the positive mesothelioma markers for this differential diagnosis. Calretinin and mesothelin can also be useful, but even though both of these markers are more sensitive than WT1, they are less specific. It should be emphasized, however, that a negative staining for both calretinin and mesothelin is strong evidence against the diagnosis of epithelioid mesothelioma. Among the group of negative markers, p63, MOC-31, Ber-EP4, CEA, and BG-8 were expressed in the large majority of squamous carcinomas, but not in mesotheliomas. p63 has the advantage over the four previously mentioned markers in that not only is it strongly and invariably expressed in squamous carcinomas, but it also may assist in distinguishing these tumors from lung adenocarcinomas which only occasionally express this marker. From a practical point-of-view, a combination of two of the positive mesothelioma markers and two of the negative ones usually allows the distinction to be made between epithelioid mesotheliomas and squamous carcinomas. Because of their specificity and sensitivity in reacting with mesotheliomas, the best combination appears to be WT1 and calretinin or mesothelin for the positive markers and p63 and MOC-31 for the negative. If lung adenocarcinoma is also included in the differential diagnosis, keratin $5 / 6$ and TTF- 1 could be added to the panel. Keratin $5 / 6$ is usually expressed in squamous carcinomas but not in lung adenocarcinomas, and TTF-1 is expressed in the large majority of lung adenocarcinomas but not in squamous carcinomas of the lung. As the novel mesothelioma marker podoplanin is often expressed in epithelioid mesotheliomas, but it is absent in lung adenocarcinomas, it can be useful in distinguishing between these tumors, but its value in discriminating between epithelioid mesotheliomas and squamous carcinomas appears to be somewhat more limited.

\section{Acknowledgements}

I thank Janet Quiñones, Raju Nandagiri, Maria Grimberg-Siehien, Kim-Anh Vu, and Mannie Steglich for technical assistance and Asuncion Moroi for secretarial assistance.

\section{References}

1 Attanoos RL, Gibbs AR. Pseudomesotheliomatous' carcinomas of the pleura: a 10-year analysis of cases from the Environmental Lung Disease Research Group, Cardiff. Histopathology 2003;43:444-452.

2 Kobashi Y, Okimoto N, Sakamoto K. Squamous cell carcinoma of the lung producing granulocyte colonystimulating factor and resembling a malignant pleural mesothelioma. Intern Med 2004;43:111-116.

3 Pritchard SA, Howat AJ, Edwards JM. Immunohistochemical panel for distinction between squamous cell carcinoma, adenocarcinoma and mesothelioma (letter). Histopathology 2003;43:197-199.

4 Attanoos RL, Thomas DH, Gibbs AR. Synchronous diffuse malignant mesothelioma and carcinomas in asbestos-exposed individuals. Histopathology 2003;43: 387-392.

5 French CA, Sugarbaker DJ, Corson JM. Synchronous pleural mesothelioma and primary lung carcinoma. Mod Pathol 1999;12:183A.

6 Miettinen M, Sarlomo-Rikala M. Expression of calretinin, thrombomodulin, keratin 5, and mesothelin in lung carcinomas of different types: an immunohistochemical analysis of 596 tumors in comparison with 
epithelioid mesotheliomas of the pleura. Am J Surg Pathol 2003;27:150-158.

7 Churg A, Roggli V, Galateau-Salle F, et al. Mesothelioma. In: Travis WD, Brambilla E, Müller-Hermelink HK, Harris CC (eds). World Health Organization Classification of Tumours. Pathology \& Genetics: Tumours of the Lung, Pleura, Thymus and Heart. IARC Press: Lyon, France, 2004, pp 128-136.

8 Ordóñez NG. The immunohistochemical diagnosis of mesothelioma: a comparative study of epithelioid mesothelioma and lung adenocarcinoma. Am J Surg Pathol 2003;27:1031-1051.

9 Ordóñez NG. Value of cytokeratin 5/6 immunostaining in distinguishing epithelial mesothelioma of the pleura from lung adenocarcinoma. Am J Surg Pathol 1998; 22:1215-1221.

10 Chu AY, Litzky LA, Pasha TL, et al. Utility of D2-40, a novel mesothelial marker, in the diagnosis of malignant mesothelioma. Mod Pathol 2005;18:105-110.

11 Kimura N, Kimura I. Podoplanin as a marker for mesothelioma. Pathol Int 2005;55:83-86.

12 Ordóñez NG. D2-40 and podoplanin are highly specific and sensitive immunohistochemical markers of epithelioid malignant mesothelioma. Hum Pathol 2005;36: 372-380.

13 Schacht V, Dadras SS, Johnson LA, et al. Up-regulation of the lymphatic marker podoplanin, a mucin-type transmembrane glycoprotein, in human squamous cell carcinomas and germ cell tumors. Am J Pathol 2005; 166:913-921.

14 Breiteneder-Geleff S, Matsui K, Soleiman A, et al. Podoplanin, novel 43-kd membrane protein of glomerular epithelial cells, is down-regulated in puromycin nephrosis. Am J Pathol 1997;151:1141-1152.

15 Sienko A, Zander DS, Killen D, et al. D2-40 is a novel new marker of malignant mesothelioma (MM): tissue microarray study of $45 \mathrm{MM}$ versus 409 lung carcinomas and primary non-mesothelial neoplasms of the pleura and chest wall. Mod Pathol 2005;18(Suppl 1):318A.

16 Doglioni C, Dei Tos AP, Laurino L, et al. Calretinin: a novel immunocytochemical marker for mesothelioma. Am J Surg Pathol 1996;20:1037-1046.

17 Gotzos V, Vogt P, Celio MR. The calcium binding protein calretinin is a selective marker for malignant pleural mesotheliomas of the epithelial type. Pathol Res Pract 1996;192:137-147.

18 Abutaily AS, Addis BJ, Roche WR. Immunohistochemistry in the distinction between malignant mesothelioma and pulmonary adenocarcinoma: a critical evaluation of new antibodies. J Clin Pathol 2002;55: 662-668.

19 Barberis MCP, Faleri M, Veronese S, et al. Calretinin: a selective marker of normal and neoplastic mesothelial cells in serous effusions. Acta Cytol 1997;41:17571761.

20 Brockstedt U, Gulyas M, Dobra K, et al. An optimized battery of eight antibodies that can distinguish most cases of epithelial mesothelioma from adenocarcinoma. Am J Clin Pathol 2000;114:203-209.

21 Carella R, Deleonardi G, D'Errico A, et al. Immunohistochemical panels for differentiating epithelial malignant mesothelioma from lung adenocarcinoma: a study with logistic regression analysis. Am J Surg Pathol 2001;25:43-50.

22 Comin CE, Novelli L, Boddi V, et al. Calretinin, thrombomodulin, CEA, and CD15: a useful combina- tion of immunohistochemical markers for differentiating pleural epithelial mesothelioma from peripheral pulmonary adenocarcinoma. Hum Pathol 2001;32: 529-536.

23 Cury PM, Butcher DN, Fisher C, et al. Value of the mesothelium-associated antibodies thrombomodulin, cytokeratin 5/6, calretinin, and $\mathrm{CD} 44 \mathrm{H}$ in distinguishing epithelioid pleural mesothelioma from adenocarcinoma metastatic to the pleura. Mod Pathol 2000; 13:107-112.

24 Davidson B, Nielsen S, Christensen J, et al. The role of desmin and N-cadherin in effusion cytology: a comparative study using established markers of mesothelial and epithelial cells. Am J Surg Pathol 2001; 25:1405-1412.

25 Leers MPG, Aarts MMJ, Theunissen PHMH. E-cadherin and calretinin: a useful combination of immunochemical markers for differentiation between mesothelioma and metastatic adenocarcinoma. Histopathology 1998;32:209-216.

26 Ordóñez NG. Value of calretinin immunostaining in differentiating epithelial mesothelioma from lung adenocarcinoma. Mod Pathol 1998;11:929-933.

27 Simsir A, Fetsch P, Abati A. Calretinin immunostaining in benign and malignant pleural effusions. Diagn Cytopathol 2001;24:149-152.

28 Tot T. The value of cytokeratins 20 and 7 in discriminating metastatic adenocarcinomas from pleural mesotheliomas. Cancer 2001;92:2727-2732.

29 Wieczorek TJ, Krane JF. Diagnostic utility of calretinin immunohistochemistry in cytologic cell block preparations. Cancer (Cancer Cytopathol) 2000;90:312-319.

30 Lugli A, Forster Y, Haas P, et al. Calretinin expression in human normal and neoplastic tissues: a tissue microarray analysis of 5233 tissue samples. Hum Pathol 2003;34:994-1000.

31 Miettinen M, Limon J, Niezabitowski A, et al. Calretinin and other mesothelioma markers in synovial sarcoma: analysis of antigenic similarities and differences with malignant mesothelioma. Am J Surg Pathol 2001;25:610-617.

32 Foster MR, Johnson JE, Olson SJ, et al. Immunohistochemical analysis of nuclear versus cytoplasmic staining of WT1 in malignant mesotheliomas and primary pulmonary adenocarcinomas. Arch Pathol Lab Med 2001;125:1316-1320.

33 Ordóñez NG. Value of thyroid transcription factor-1, E-cadherin, BG8, WT1, and CD44S immunostaining in distinguishing epithelial pleural mesothelioma from pulmonary and nonpulmonary adenocarcinoma. Am J Surg Pathol 2000;24:598-606.

34 Amin KM, Litzky LA, Smythe WR, et al. Wilms' tumor 1 susceptibility (WT1) gene products are selectively expressed in malignant mesothelioma. Am J Pathol 1995;146:344-356.

35 Kumar-Singh S, Segers K, Rodeck U, et al. WT1 mutation in malignant mesothelioma and WT1 immunoreactivity in relation to p53 and growth factor receptor expression, cell-type transition, and prognosis. J Pathol 1997;181:67-74.

36 Yaziji H, Battifora H, Bacehi CD, et al. Definitive diagnosis of pleural mesothelioma can be established on a small panel of immunohistochemical markers using logic regression, a novel statistical method. Med Pathol 2003;16:316A.

37 Hwang H, Quenneville L, Yaziji H, et al. Wilms tumor gene product: sensitive and contextually specific 
marker of serous carcinomas of ovarian surface epithelial origin. Appl Immunohistochem Mol Morphol 2004;12:122-126.

38 Chang K, Pai LH, Batra JK, et al. Characterization of the antigen (CAK1) recognized by monoclonal antibody K1 present on ovarian cancers and normal mesothelium. Cancer Res 1992;52:181-186.

39 Chang K, Pai LH, Pass H, et al. Monoclonal antibody K1 reacts with epithelial mesothelioma but not with lung adenocarcinoma. Am J Surg Pathol 1992;16:259-268.

40 Ordóñez NG. Value of mesothelin immunostaining in the diagnosis of mesothelioma. Mod Pathol 2003;16: 192-197.

41 Yang A, Kaghad M, Wang Y, et al. p63, a p53 homolog at 3q27-29, encodes multiple products with transactivating, death-inducing, and dominant-negative activities. Mol Cell 1998;2:305-316.

42 Reis-Filho JS, Schmitt FC. Taking advantage of basic research: p63 is a reliable myoepithelial and stem cell marker. Adv Anat Pathol 2002;9:280-289.

$43 \mathrm{Au}$ NHC, Gown AM, Cheang M, et al. p63 expression in lung carcinoma: a tissue microarray study of 408 cases. Appl Immunohistochem Mol Morphol 2004; 12:240-247.

44 Kaufmann O, Fietze E, Mengs J, et al. Value of p63 and cytokeratin 5/6 as immunohistochemical markers for the differential diagnosis of poorly differentiated and undifferentiated carcinomas. Am J Clin Pathol 2001; 116:823-830.

45 Reis-Filho JS, Simpson PT, Martins A, et al. Distribution of p63, cytokeratins 5/6 and cytokeratin 14 in 51 normal and 400 neoplastic human tissue samples using TARP-4 multi-tumor tissue microarray. Virchows Arch 2003;443:122-132.

$46 \mathrm{Wu} \mathrm{M}$, Wang $\mathrm{B}$, Gil J, et al. p63 and TTF-1 immunostaining: a useful marker panel for distinguishing small cell carcinoma of lung from poorly differentiated squamous cell carcinoma of lung. Am J Clin Pathol 2003;119:696-702.

47 Zhang H, Liu J, Cagle PT, et al. Distinction of pulmonary small cell carcinoma from poorly differentiated squamous cell carcinoma: an immunohistochemical approach. Mod Pathol 2005;18:111-118.

48 Pan CC, Chen PC, Chou TY, et al. Expression of calretinin and other mesothelioma-related markers in thymic carcinoma and thymoma. Hum Pathol 2003; 34:1155-1162.

49 Sheikh HA, Fuhrer K, Cieply K, et al. p63 expression in assessment of bronchioloalveolar proliferations of the lung. Mod Pathol 2004;17:1134-1140.

50 Oates J, Edwards C. HBME-1, MOC-31, WT1 and calretinin: as assessment of recently described markers for mesothelioma and adenocarcinoma. Histopathology 2000;36:341-347.

51 Ordóñez NG. Value of the MOC-31 monoclonal antibody in differentiating epithelial pleural mesothelioma from lung adenocarcinoma. Hum Pathol 1998;29: 166-169.

52 Ruitenbeek T, Gouw ASH, Poppema S. Immunocytology of body cavity fluids: MOC-31, a monoclonal antibody discriminating between mesothelial and epithelial cells. Arch Pathol Lab Med 1994;118: 265-269.

53 Winter MJ, Nagtegaal ID, van Krieken JHJM, et al. The epithelial cell adhesion molecule (Ep-CAM) as a morphoregulatory molecule is a tool in surgical pathology. Am J Pathol 2003;163:2139-2148.
54 Latza U, Niedobitek G, Schwarting R, et al. Ber-EP4: new monoclonal antibody which distinguishes epithelia from mesothelia. J Clin Pathol 1990;43:213-219.

55 Ordóñez NG. Value of the Ber-EP4 antibody in differentiating epithelial pleural mesothelioma from adenocarcinoma. The M.D. Anderson experience and a critical review of the literature. Am J Clin Pathol 1998;109:85-89.

56 Maguire B, Whitaker D, Carrello S, et al. Monoclonal antibody Ber-EP4: its use in the differential diagnosis of malignant mesothelioma and carcinoma in cell blocks of malignant effusions and FNA specimens. Diagn Cytopathol 1994;10:130-134.

57 Jordon D, Jagirdar J, Kaneko M. Blood group antigens, Lewis $^{\mathrm{x}}$ and Lewis ${ }^{\mathrm{y}}$, in the diagnostic discrimination of malignant mesothelioma versus adenocarcinoma. Am J Pathol 1989;135:931-937.

58 Riera JR, Astengo-Osuna C, Longmate JA, et al. The immunohistochemical diagnositc panel for epithelial mesothelioma. A reevaluation after heat-induced epitope retrieval. Am J Surg Pathol 1997;21:1409-1419.

59 Lyda MH, Weiss LM. Immunoreactivity for epithelial and neuroendocrine antibodies are useful in the differential diagnosis of lung carcinomas. Hum Pathol 2000;31:980-987.

60 Sheibani K, Battifora H, Burke JS. Antigenic phenotype of malignant mesotheliomas and pulmonary adenocarcinomas: an immunohistologic analysis demonstrating the value of Leu M1 antigen. Am J Pathol 1986;123: 212-219.

61 Kadota A, Masutani M, Takei M, et al. Evaluation of expression of CD15 and sCD15 in non-small cell lung cancer. Int J Oncol 1999;15:1081-1089.

62 Spagnolo DV, Whitaker D, Carrello S, et al. The use of monoclonal antibody $44-3 \mathrm{~A} 6$ in cell blocks in the diagnosis of lung carcinoma, carcinomas metastatic to lung and pleura, and pleural malignant mesothelioma. Am J Clin Pathol 1991;95:322-329.

63 Ando S, Kimura H, Iwai N, et al. Optimal combination of seven tumour markers in prediction of advanced stage at first examination of patients with non-small cell lung cancer. Anticancer Res 2001;21:3085-3092.

64 Johnston WW. Applications of monoclonal antibodies in clinical cytology as exemplified by studies with monoclonal antibody B72.3. Acta Cytol 1987;31: 537-556.

65 López-Ferrer A, Barranco C, de Bolós C. Differences in the $O$-glycosylation patterns between lung squamous cell carcinoma and adenocarcinoma. Am J Clin Pathol 2002;118:749-755.

66 Loy TS, Nashelsky MB. Reactivity of B72.3 with adenocarcinomas: an immunohistochemical study of 476 cases. Cancer 1993;7:2495-2498.

67 Ordóñez NG. Thyroid transcription factor-1 is a marker of lung and thyroid carcinomas. Adv Anat Pathol 2000;7:123-127.

68 Di Loreto C, Di Lauro V, Puglisi F, et al. Immunocytochemical expression of tissue specific transcription factor-1 in lung carcinoma. J Clin Pathol 1997;50: 30-32.

69 Fabbro D, Di Loreto C, Stamerra O, et al. TTF-1 gene expression in human lung tumours. Eur J Cancer 1996;32A:512-517.

70 Puglisi F, Barbone F, Damante G, et al. Prognostic value of thyroid transcription factor-1 in primary, resected, non-small cell lung carcinoma. Mod Pathol 1999;12: 318-324. 
71 Chang Y-L, Lee Y-C, Liao W-Y, et al. The utility and limitation of thyroid transcription factor-1 protein in primary and metastatic pulmonary neoplasms. Lung Cancer 2004;44:149-157.

72 Jerome Marson V, Mazieres J, Groussard O, et al. Expression of TTF-1 and cytokeratins in primary and secondary epithelial lung tumours: correlation with histological type and grade. Histopathology 2004;45: 125-134.

73 Khoor A, Whitsett JA, Stahlman MT, et al. Utility of surfactant protein B precursor and thyroid transcription factor 1 in differentiating adenocarcinoma of the lung from malignant mesothelioma. Hum Pathol 1999;30:695-700.

74 Nakamura N, Miyagi E, Murata S, et al. Expression of thyroid transcription factor-1 in normal and neoplastic lung tissues. Mod Pathol 2002;15:1058-1067.

75 Sturm N, Lantuéjoul S, Laverrière M-H, et al. Thyroid transcription factor 1 and cytokeratins 1, 5, 10, 14 (34 $\beta$ E12) expression in basaloid and large-cell neuroendocrine carcinomas of the lung. Hum Pathol 2001;32:918-925.

76 Baars JH, De Ruijter JLM, Smedts F, et al. The applicability of a keratin 7 monoclonal antibody in routinely Papanicolaou-stained cytologic specimens for the differential diagnosis of carcinomas. Am J Clin Pathol 1994;101:257-261.

77 Chu P, Wu E, Weiss LM. Cytokeratin 7 and cytokeratin 20 expression in epithelial neoplasms: a survey of 435 cases. Mod Pathol 2000;13:962-972.

78 Fukai I, Masaoka A, Hashimoto T, et al. Differential diagnosis of thymic carcinoma and lung carcinoma with the use of antibodies to cytokeratins. J Thorac Cardiovasc Surg 1995;110:1670-1675.

79 Pomplun S, Wotherspoon AC, Shah G, et al. Immunohistochemical markers in the differentiation of thymic and pulmonary neoplasms. Histopathology 2002;40: 152-158.

80 van de Molengraft FJJM, van Niekerk CC, Jap PHK, et al. OV-TL 12/30 (keratin 7 antibody) is a marker of glandular differentiation in lung cancer. Histopathology 1993;22:35-38.

81 Wang NP, Zee S, Zarbo RJ, et al. Coordinate expression of cytokeratins 7 and 20 defines unique subsets of carcinomas. Appl Immunohistochem 1995;3:99-107.

82 Johansson L. Histopathologic classification of lung cancer: relevance of cytokeratin and TTF-1 immunophenotyping. Ann Diagn Pathol 2004;8:259-267. 